The Astronomical Journal, 122:1429-1437, 2001 September

\title{
ABUNDANCES IN STARS FROM THE RED GIANT BRANCH TIP TO NEAR THE MAIN-SEQUENCE TURNOFF IN M71. II. IRON ABUNDANCE ${ }^{1}$
}

\author{
Solange V. Ramírez, ${ }^{2}$ Judith G. Cohen, ${ }^{2}$ Jeremy Buss, ${ }^{3}$ ANd Michael M. Briley ${ }^{3}$ \\ Received 2001 March 29; accepted 2001 May 18
}

\begin{abstract}
We present $[\mathrm{Fe} / \mathrm{H}]$ abundance results that involve a sample of stars with a wide range in luminosity, from luminous giants to stars near the turnoff in a globular cluster. Our sample of 25 stars in M71 includes 10 giant stars more luminous than the RHB, three horizontal branch stars, nine giant stars less luminous than the RHB, and three stars near the turnoff. We analyzed both $\mathrm{Fe} I$ and $\mathrm{Fe}$ II lines in high-dispersion spectra observed with HIRES at the W. M. Keck Observatory. We find that the $[\mathrm{Fe} / \mathrm{H}]$ abundances from both $\mathrm{Fe} \mathrm{I}$ and $\mathrm{Fe}$ II lines agree with each other and with earlier determinations. Also the $[\mathrm{Fe} / \mathrm{H}]$ obtained from $\mathrm{Fe} \mathrm{I}$ and $\mathrm{Fe}$ II lines is constant within the rather small uncertainties for this group of stars over the full range in $T_{\text {eff }}$ and luminosity, suggesting that non-LTE effects are negligible in our iron abundance determination. In this globular cluster, there is no difference among the mean $[\mathrm{Fe} / \mathrm{H}]$ of giant stars located at or above the RHB, RHB stars, giant stars located below the RHB and stars near the turnoff.
\end{abstract}

Key words: globular clusters: general — globular clusters: individual (M71) — stars: abundances stars: evolution

\section{INTRODUCTION}

Abundance determinations of stars in Galactic globular clusters can provide valuable information about important astrophysical processes, such as stellar evolution, stellar structure, Galactic chemical evolution, and the formation of the Milky Way. Surface stellar abundances of C, N, O, and often $\mathrm{Na}, \mathrm{Mg}$, and $\mathrm{Al}$ are found to be variable among red giants within a globular cluster. The physical process responsible of these star-to-star element variations is still uncertain (see Cohen, Briley, \& Behr 2001, Paper I).

Of particular importance to the present study are the results of King et al. (1998), who found that $[\mathrm{Fe} / \mathrm{H}]$ among M92 subgiants is a factor of 2 smaller than $[\mathrm{Fe} / \mathrm{H}]$ from red giants in the same cluster (Cohen 1978; Sneden et al. 1991). If this result is not due to systematic differences arising from the analysis procedures of the different groups that handle the red giant branch (RGB) and the subgiant samples, then the results of King et al. (1998) would suggest some modification of photospheric $\mathrm{Fe}$ abundances that would be quite difficult to explain. Clearly the present day cluster stars are incapable of modifying their own Fe abundances through nucleosynthesis. Yet evolution-driven changes in $[\mathrm{Fe} / \mathrm{H}]$ could be possible if the outer envelopes of the subgiants were somehow infused with Fe-poor external material. Thus as evolution progresses up the RGB the deepening convective envelope would dilute the photospheric $[\mathrm{Fe} / \mathrm{H}]$ with more $\mathrm{Fe}$-rich " unmodified" material from the interior, causing an increase in $[\mathrm{Fe} / \mathrm{H}]$ with luminosity. We note the unlikelihood of this scenario, as $\mathrm{Fe}$, unlike the lighter elements, is not involved in normal mass transfer processes

\footnotetext{
${ }^{1}$ Based on observations obtained at the W. M. Keck Observatory, which is operated as a scientific partnership among the California Institute of Technology, the University of California, and the National Aeronautics and Space Administration. The Observatory was made possible by the generous financial support of the W. M. Keck Foundation.

2 Palomar Observatory, Mail Stop 105-24, California Institute of Technology, Pasadena, CA 91125.

${ }^{3}$ Department of Physics, University of Wisconsin, Oshkosh, Oshkosh, WI 54901.
}

that might occur in binary systems or in normal stellar winds and is only produced in supernovae. Moreover, it is difficult to understand how cluster stars could be contaminated by material with less $\mathrm{Fe}$, presumably at a time after the present subgiants had formed.

In order to study the origin of the star-to-star abundance variations and to address the issues raised by King et al. (1998), we have started a program to determine chemical abundances of the nearer Galactic globular cluster stars. In this paper, we present our results for the iron abundance of M71, the nearest globular cluster reachable from the northern hemisphere. Similar programs are underway at ESO by Castilho et al. (2000) and Gratton et al. (2001), taking advantage of the fact that the nearest globular cluster accessible from a southern site (NGC 6397) is 1.8 times closer than M71 and has a lower reddening. Our M71 sample includes stars over a large range in luminosity: 19 giant stars, three horizontal branch stars, and three stars near the main sequence turnoff, in order to study in a consistent manner red giants, horizontal branch stars, and stars near the main-sequence turnoff. Details on the star sample, observations, data reduction, and determination of stellar parameters are described in Paper I. Previous highdispersion abundance analysis for M71 involves studies of red giants only, resulting in $[\mathrm{Fe} / \mathrm{H}]$ of -0.70 (Cohen 1983), -0.80 (Gratton, Quarta, \& Ortolani 1986), between -0.6 and -1.0 (Leep, Oke, \& Wallerstein 1987), and -0.79 (Sneden et al. 1994).

\section{ANALYSIS}

We begin our analysis with $\mathrm{Fe}$, as many $\mathrm{Fe}$ lines are identified in our HIRES spectra over a wide range of excitation potentials and line strengths, as well as over two ionization states ( $\mathrm{Fe} \mathrm{I}$ and $\mathrm{Fe}$ II). The iron abundance analysis is done using a current version of the LTE spectral synthesis program MOOG (Sneden 1973). A line list specifying the wavelengths, excitation potentials, $g f$-values, damping constants, and equivalent widths for the observed $\mathrm{Fe}$ I and $\mathrm{Fe}$ II lines is required. The provenance of the $g f$-values and the measurement of equivalent widths are discussed below. The 
damping constants for all $\mathrm{Fe} \mathrm{I}$ and $\mathrm{Fe}$ II lines were set to twice that of the Unsöld approximation for van der Waals broadening following Holweger et al. (1991). The use of the Blackwell approximation for the damping constants gives the same result within the errors, when comparing $[\mathrm{Fe} / \mathrm{H}]$ obtained with the "good-line set" of $\mathrm{Fe}$ I (see definition of line sets on $\S 2.2$ ).

In addition, a model atmosphere for the effective temperature and surface gravity appropriate for each star and a value for the microturbulent velocity are also required. We use the grid of model atmospheres from Kurucz (1993b) with a metallicity of $[\mathrm{Fe} / \mathrm{H}]=-0.5$ dex, based on earlier high-dispersion abundance analysis of M71 red giants (Cohen 1983; Gratton et al. 1986; Leep et al. 1987; Sneden et al. 1994). The final result for $[\mathrm{Fe} / \mathrm{H}]$ is not sensitive to small changes in the metallicity of the model atmosphere. In particular, the error introduced by using a model with $[\mathrm{Fe} / \mathrm{H}]=-0.5$ instead of $-0.7 \mathrm{dex}$ is very small (see Tables 2 and 3). The effective temperatures and surface gravities are derived from the photometry of the stars as described in Paper I. The error in the photometric $T_{\text {eff }}$ is 75 $\mathrm{K}$ for giants and $150 \mathrm{~K}$ for the dwarfs and the error in the photometric $\log (g)$ is 0.2 dex (Paper I). The microturbulent velocity is derived spectroscopically (see below). The stellar parameters are listed in Table 1.

\subsection{Transition Probabilities}

Transition probabilities for the $\mathrm{Fe}$ I lines were obtained from several laboratory experiments, including studies of Fe I absorption lines produced by iron vapor in a carbon tube furnace (Blackwell, Petford, \& Shallis 1979; Blackwell et al. 1982a; Blackwell, Petford, \& Simmons 1982b; Blackwell et al. 1986) (Oxford Group), measurement of radiative lifetimes of $\mathrm{Fe} I$ transitions by laser induced fluorescence (O’Brian et al. 1991; Bard, Kock, \& Kock 1991; Bard \& Kock 1994), Fe I emission-line spectroscopy from a lowcurrent arc (May, Richter, \& Wichelmann 1974), and emission lines of $\mathrm{Fe}$ I from a shock tube (Wolnik, Berthel, \& Wares 1971). We also considered solar $g f$-values from Thévenin $(1989,1990)$ when needed.

We compare the $g f$-values obtained by the different experiments in an attempt to place them onto a common scale with respect to the results from O'Brian et al. (1991), who provided the longest list of $g f$-values. We considered for the comparison only the set of lines present in our data, which have a wavelength coverage roughly from 5380 to $7900 \AA$. We found that the values of O'Brian et al. (1991) and of the Oxford Group were on the same scale; the mean difference in $\log (g f)$ between the two experiments is $0.02 \pm 0.01$ for 21 lines in common. Similar results are found when considering 21 lines in common between O’Brian et al. (1991) and Bard et al. (1991) (see also Bard \& Kock 1994), where the mean difference in $\log (g f)$ is $0.02 \pm 0.04$. Considering 34 lines in common between O'Brian et al. (1991) and May et al. (1974), the mean difference in $\log (g f)$ is $0.03 \pm 0.03$. When comparing the 14 lines in common between O'Brian et al. (1991) and Wolnik et al. (1971) we found a mean difference in $\log (g f)$ of $-0.07 \pm 0.02$. We also compared the results from O'Brian et al. (1991) with solar $g f$-values obtained by Thévenin $(1989,1990)$. We found that the mean difference in $\log (g f)$ is $+0.05 \pm 0.02$ when comparing 68 lines in common between these latest works. Similar offsets are found by Lambert et al. (1996).
The $g f$-values for our $\mathrm{Fe}$ I lines were taken when possible from laboratory data in the following order of priority: from O'Brian et al. (1991), from the Oxford Group, from May et al. (1974), and from Wolnik et al. (1971) corrected by -0.07 dex. In the cases where no laboratory data were available, we used solar $g f$-values from Thévenin (1989, 1990) corrected by +0.05 dex.

Transition probabilities for the Fe II lines were taken from the solar analysis of Blackwell, Shallis, \& Simmons (1980), Biémont et al. (1991), and from the semiempirical calculations of Kurucz (1993b). While restricting ourselves to lines present in our spectra, we compared the $g f$-values from Blackwell et al. (1980) and Kurucz (1993b) with the study of Biémont et al. (1991). There are nine lines in common between Biémont et al. (1991) and Blackwell et al. (1980). The mean difference between the two in $\log (g f)$ is $0.14 \pm 0.02$. A similar offset was found by Lambert et al. (1996). We found no significant difference between the results of Biémont et al. (1991) and Kurucz (1993b), since the nine lines in common result in a mean difference of $0.03 \pm 0.02$.

The $g f$-values for our $\mathrm{Fe}$ II lines were taken in the following order of priority: from Biémont et al. (1991), from Blackwell et al. (1980) corrected by 0.14 dex, and from Kurucz (1993b).

\subsection{Measurement of Equivalent Widths}

Our sample contains many stars observed in multiple orders, with many detectable absorption features in these high signal-to-noise ratio spectra. For example, in the coolest M71 star in our sample, M71-1-45, 1407 absorption lines have been identified. A FORTRAN code to automatically search for absorption features and measure their equivalent width $\left(W_{\lambda}\right)$, EWDET, was developed for this project. The code is available upon request to S. V. R. EWDET determines the continuum location of the HIRES spectra by fitting a curve to the spectra, performing several iterations of point rejection above and below sigma levels given by the user. Then EWDET identifies lines above the noise level defined by a factor of 2 of the continuum rms dispersion. Each of the identified lines is fit by a Gaussian profile and then the $W_{\lambda}$ are computed by the integration of the fitted Gaussian. The error in $W_{\lambda}$ is computed by adding quadratically the error at each point of integration, $\sigma_{i}$, times the step of the integration. The error at each point of integration is given by

$$
\begin{aligned}
\sigma_{i}^{2}= & g\left(\lambda_{i}\right)^{2} \times\left[\frac{\sigma_{P}^{2}}{P^{2}}+\frac{\left(\lambda_{i}-\lambda_{\text {cen }}\right)^{2}}{\sigma^{4}} \sigma_{\lambda_{\text {cen }}}^{2}\right. \\
& \left.+\frac{\left(\lambda_{i}-\lambda_{\text {cen }}\right)^{4}}{\sigma^{6}} \sigma_{\sigma}^{2}+\sigma_{\text {cont }}^{2}\right],
\end{aligned}
$$

where $g\left(\lambda_{i}\right)$ is the Gaussian profile, $P$ is the peak of the Gaussian, $\sigma_{P}$ is the error in the peak of the Gaussian, $\lambda_{\text {cen }}$ is the central wavelength of the Gaussian, $\sigma_{\lambda_{\text {cen }}}$ is the error in the central wavelength, $\sigma$ is the dispersion of the Gaussian, $\sigma_{\sigma}$ is the error in the dispersion of the Gaussian, and $\sigma_{\text {cont }}$ is the error in the continuum. The errors of the Gaussian parameters are from the covariance matrix of the Gaussian fit. The expression for the error in $W_{\lambda}$ is deduced by propagating the errors of the Gaussian parameters and assuming that the continuum level is equal to one (see the Appendix). The fit by a Gaussian profile is reasonable even for the strongest lines we use, as shown in Figure 1, where the 
TABLE 1

Stellar PARAMETERS For THE M71 SAMPLe

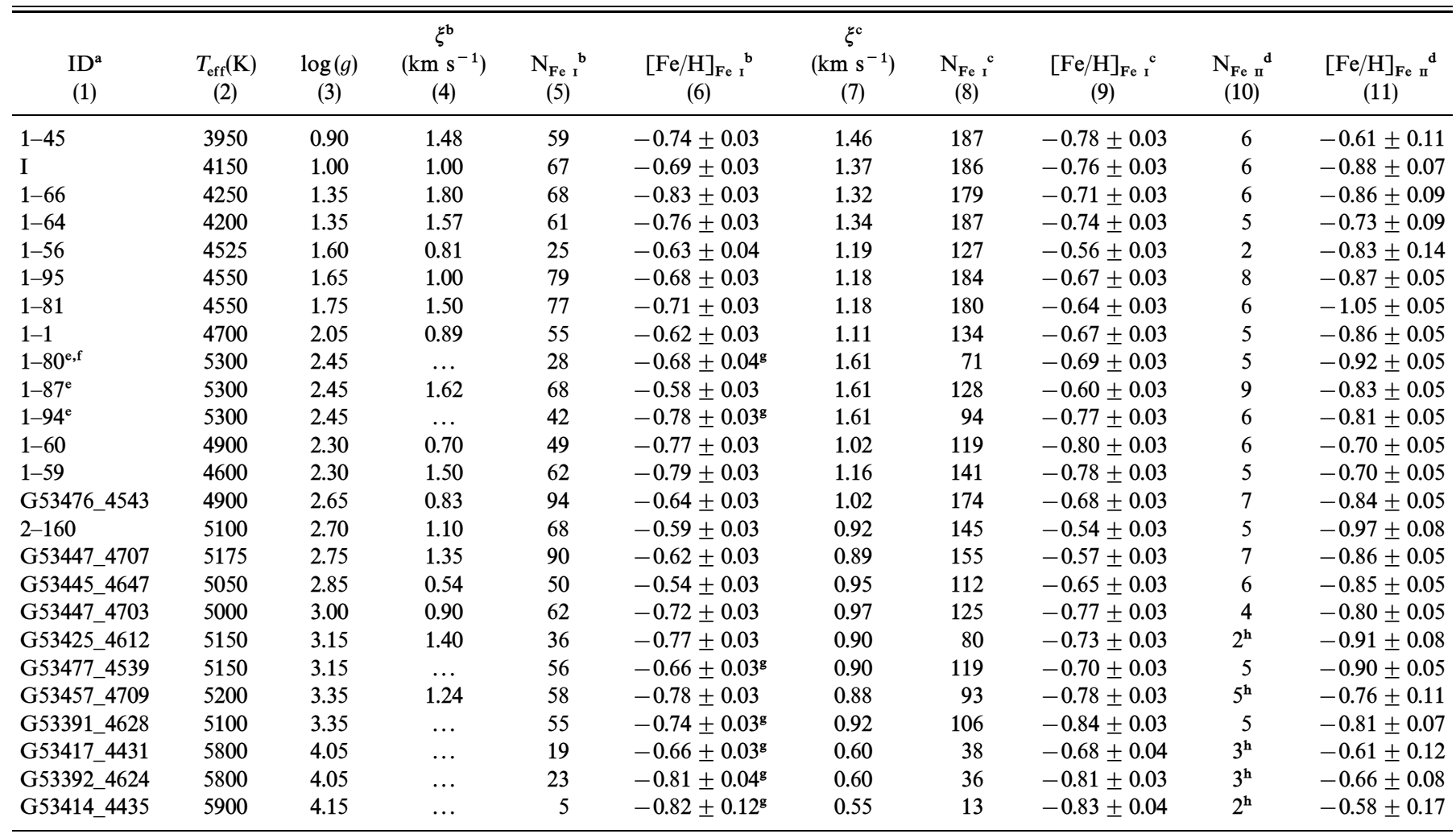

a Identifications are from Arp \& Hartwick (1971) or are assigned based on the J2000.0 coordinates, rh rm rs.s dd dm dd becoming Grmrss_dmdd.

b Set of weak Fe I lines.

${ }^{\mathrm{c}}$ Set of all good $\mathrm{Fe}$ I lines.

${ }^{\mathrm{d}} \mathrm{Set}$ of all good $\mathrm{Fe}$ II lines.

e RHB star.

${ }^{\mathrm{f}}$ Appears to show rotation (Paper I).

g Computed with $\xi$ from the set of all good $\mathrm{Fe}$ I lines.

${ }^{\mathrm{h}}$ Includes additional $\mathrm{Fe}$ II lines selected by hand.

observed line is plotted with a solid curve and its corresponding Gaussian profile fit is shown with a dashed curve.

The line list identified and measured by EWDET is then correlated to the line list with the atomic parameters to specifically identify the $\mathrm{Fe} \mathrm{I}$ and $\mathrm{Fe}$ II lines. The detailed lists of $W_{\lambda}$ and $g f$-values will be given in the next paper.

The spectral resolution $\lambda / \Delta \lambda$ of an echelle is fixed, unlike a low incidence, angle of low order grating spectrograph, where $\Delta \lambda$ is constant, independent of $\lambda$. Hence a line of constant central depth $D$ will have an equivalent width proportional to $\lambda D$. We construct the relationship between $\lambda D$ and $W_{\lambda}$ for the $\mathrm{Fe}$ I lines to look for possible blends and for saturation effects. The $\lambda D$ versus $W_{\lambda}$ relation for three stars is plotted in Figure 2; M71-I is one of the most luminous and coolest stars of our sample, M71-G53476_4543 is a medium-luminosity and temperature star, and M71G53392 4624 is one of the faintest and hottest stars of the sample. We fit a second-order polynomial to the $\lambda D-W_{\lambda}$ relationship for each star, performing several iterations of point rejection above and below the $2 \sigma$ level. The secondorder term is needed to account for line saturation at large $W_{\lambda}$, and the rejection of points is needed to eliminate blended lines. The second order fit is plotted as a solid line in Figure 2, and the points considered for such a fit are shown in black.

For Fe I, we use two sets of lines. The first set, subsequently called "the weak line set," contains those Fe I lines which are within $2 \sigma$ levels of the $\lambda D-W_{\lambda}$ fit, have $W_{\lambda}<60$ $\mathrm{m} \AA$, and have errors less than a third of the $W_{\lambda}$. This set of lines produces a sample of the best weak $\mathrm{Fe} I$ lines with the most accurate $W_{\lambda}$ and the abundances derived using them will have a minimal dependence on the choice of microturbulent velocity. The second set, subsequently called "the good-line set," consists of all the $\mathrm{Fe}$ I lines with errors less than a third of the $W_{\lambda}$ and with $W_{\lambda}$ computed from the fit for $\lambda D-W_{\lambda}$ determined for each star. In future papers, the $W_{\lambda}$ of lines of other elements will be determined in the same manner as the $\mathrm{Fe} \mathrm{I}$ lines of the good-line set. This way a consistent comparison can be done among the resulting abundances without a restriction on the strength of the lines used. The weak-line set is different for each star. Actually the weak lines for the stars near the main sequence are no longer weak for the cooler stars in our sample. We compare the results from 20 lines common with 15 stars over almost the whole range in $T_{\text {eff }}$ with the results from the weak-line set; there is no difference within the errors, nor a trend in $T_{\text {eff }}$.

For Fe II, the $W_{\lambda}$ of the lines are also determined using the fit to the $\lambda D-W_{\lambda}$ relation of the Fe I lines of the good-line set. The $\mathrm{Fe}$ II lines follow the relationship determined from $\mathrm{Fe}$ I lines well, as shown in Figure 3. Additional Fe II lines, not picked up automatically, were measured by hand for the stars near the turnoff and for M71-G53425_4612 and M71G53457_4709. The set of $W_{\lambda}$ for the hand-selected Fe II lines 

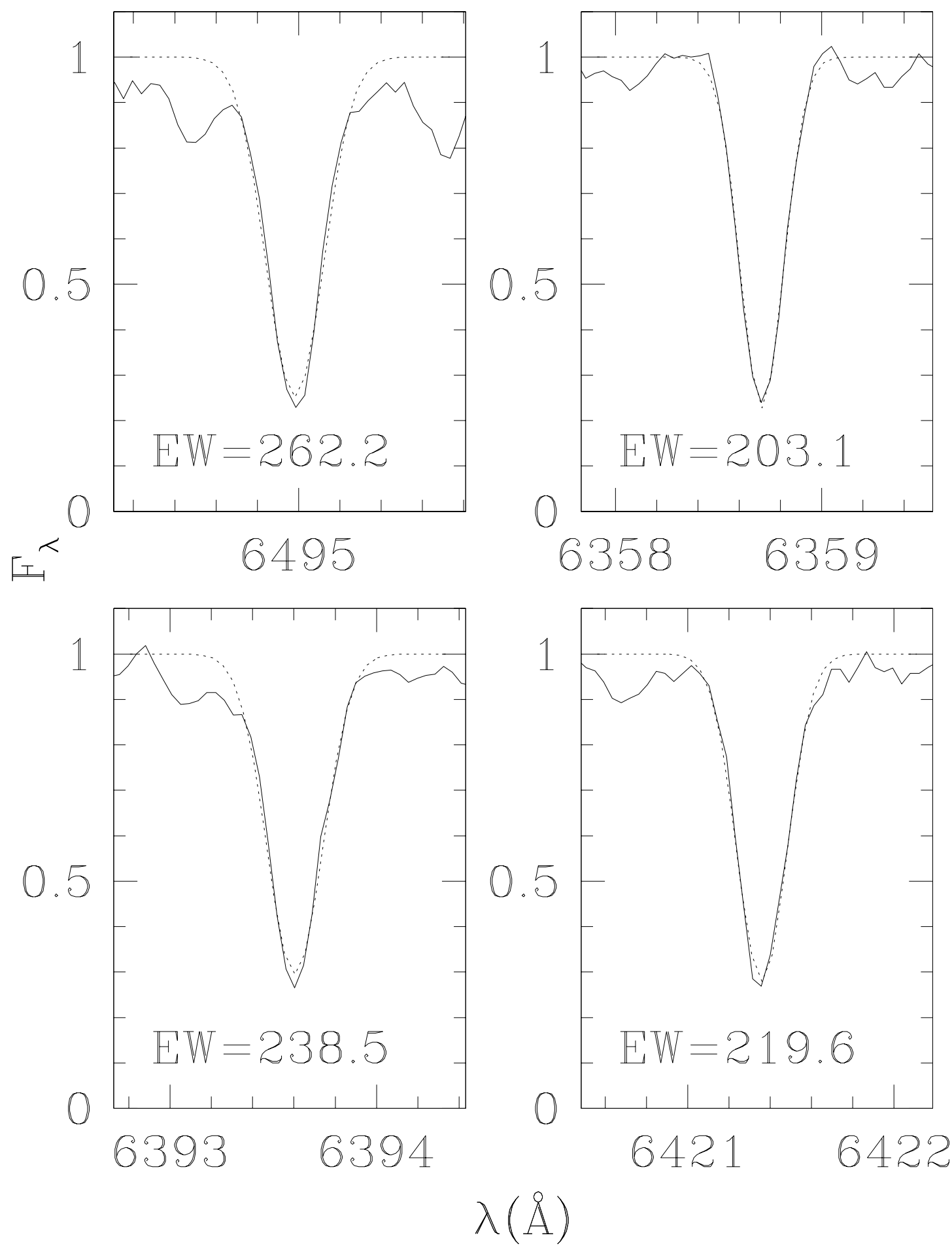

FIG. 1.- Strongest observed Fe I lines for M71-1-45. The observed lines are plotted with a solid line, and the corresponding Gaussian profile is plotted with a dashed line. The $W_{\lambda}$ of each line is indicated in the bottom left hand corner of each panel.

is computed from the $\lambda D-W_{\lambda}$ relation of the Fe I lines, after determining their observed depth from the spectra.

The number of $\mathrm{Fe} I$ lines, for both sets of lines and the number of $\mathrm{Fe}$ II lines utilized in this analysis are listed in Table 1.

\subsection{Spectroscopic Effective Temperature}

The effective temperature ( $\left.T_{\text {eff }}\right)$ of a star can be determined spectroscopically by requiring the abundance to be independent of the lower excitation potential. This tech- nique can be applied to 20 of our stars where we have Fe I lines with enough range in lower excitation potential to do so. For the spectroscopic $T_{\text {eff }}$ determination we are using the weak-line set of Fe I lines, because its resulting abundance and spectroscopic $T_{\text {eff }}$ will be only weakly dependent on the choice of microturbulent velocity. We find that the spectroscopic $T_{\text {eff }}$ is in good agreement with the photometric $T_{\text {eff }}$ derived in Paper I, as shown in Figure 4. The solid line in Figure 4 shows the ideal case when the spectroscopic and the photometric $T_{\text {eff }}$ are equal. The scatter 


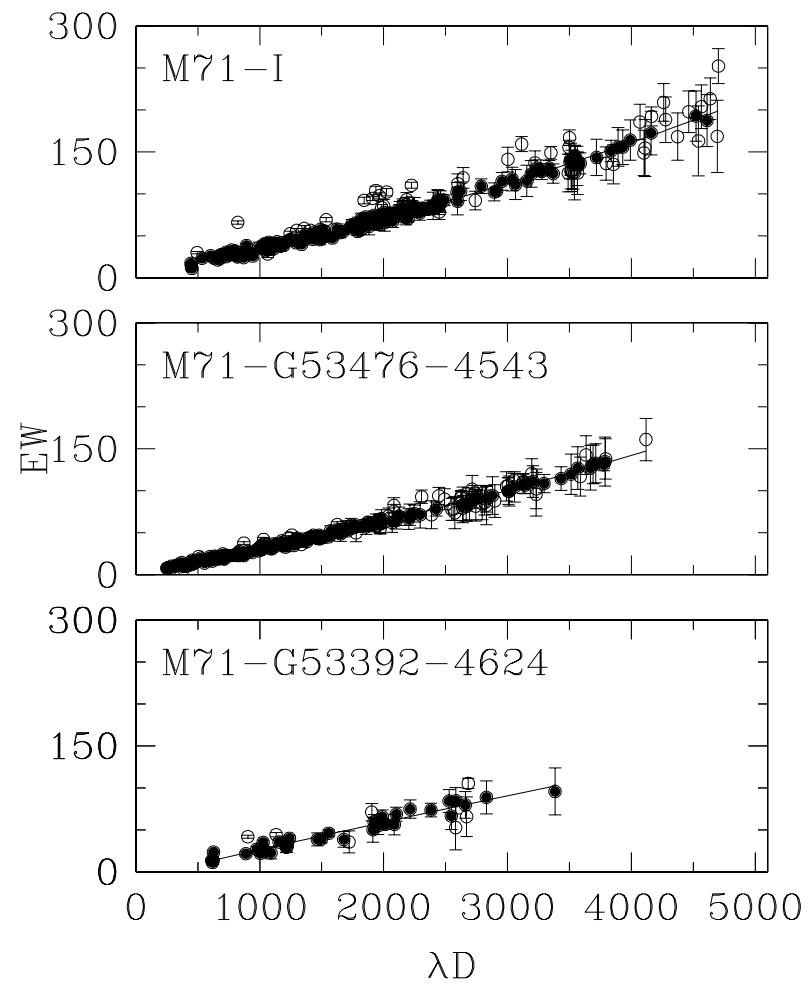

FIG. 2.-Central depth times wavelength vs. $W_{\lambda}$ relations for M71-I (one of the most luminous and coolest stars in our sample), M71G53476_4543 (a star of medium luminosity and temperature), and M71G53392_4624 (one of the faintest and hottest stars in our sample). The solid curve is a second-order fit obtained after several iterations of rejection of points deviating by $2 \sigma$ or more. The points used in the fit are shown in black.

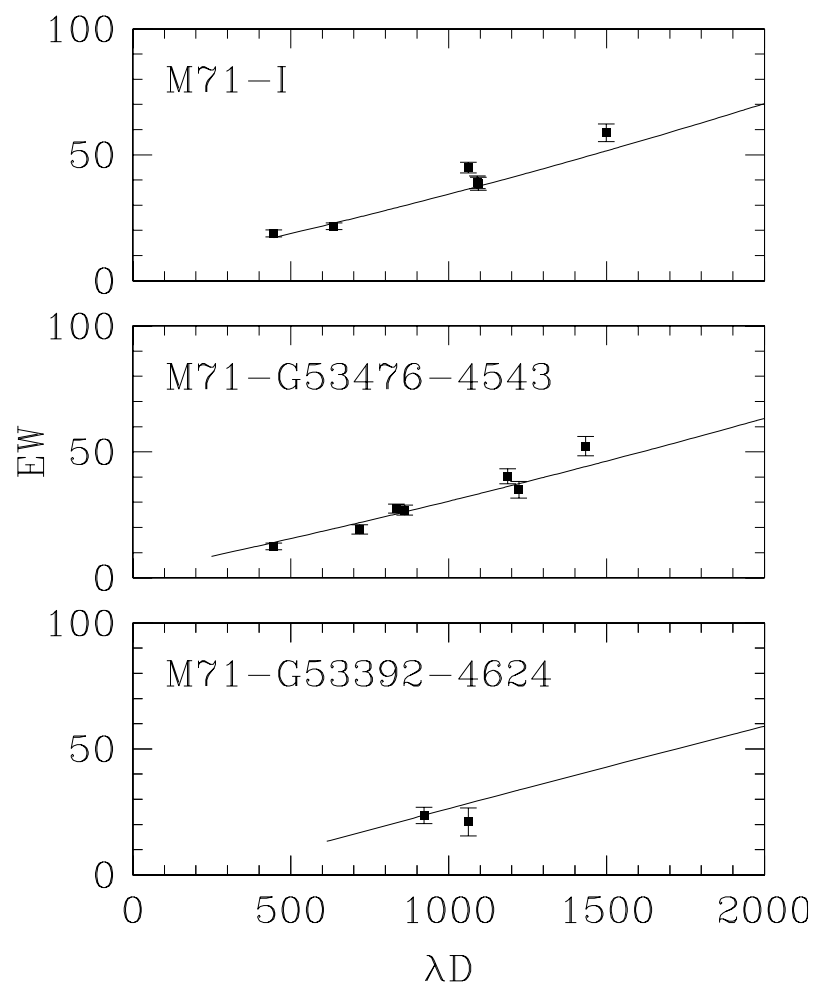

Fig. 3.-Central depth times wavelength vs. $W_{\lambda}$ relations for M71-I (one of the most luminous and coolest stars in our sample), M71G53476_4543 (a star of medium luminosity and temperature), and M71G53392_4624 (one of the faintest and hottest stars in our sample). Solid squares denote identified $\mathrm{Fe}$ II lines, and the solid curve is the second-order fit obtained for $\mathrm{Fe} \mathrm{I}$ lines and shown in Fig. 2.

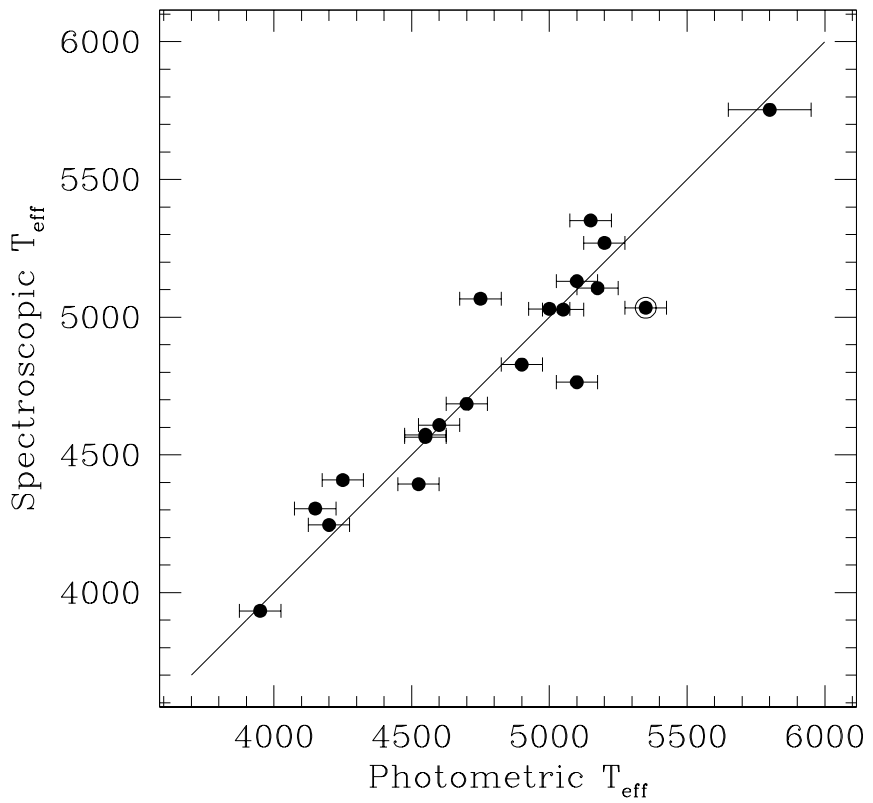

FIG. 4.-Photometric $T_{\text {eff }}$ vs. spectroscopic $T_{\text {eff }}$ for the M71 sample. The solid line indicates the ideal case when the photometric and spectroscopic $T_{\text {eff }}$ have the same value. The scatter around the solid line is about $150 \mathrm{~K}$. The only RHB star for which $T_{\text {eff }}$ can be determined spectroscopically is marked with an open circle.

around the solid line is about $150 \mathrm{~K}$, which is comparable to the error of the photometric $T_{\text {eff }}$ of $75 \mathrm{~K}$ for giants and of $150 \mathrm{~K}$ for dwarfs (Paper I), also shown in Figure 4. The scatter around the solid line is symmetric, not above or below, indicating the lack of systematic effects with the photometric temperatures.

\subsection{Microturbulent Velocity}

The microturbulent velocity $(\xi)$ of a star can be determined spectroscopically by requiring the abundance to be independent of the strength of the lines measured as the equivalent width. We apply this technique for both sets of $\mathrm{Fe}$ I lines. The resulting $\xi$ and the $[\mathrm{Fe} / \mathrm{H}]$ computed with it

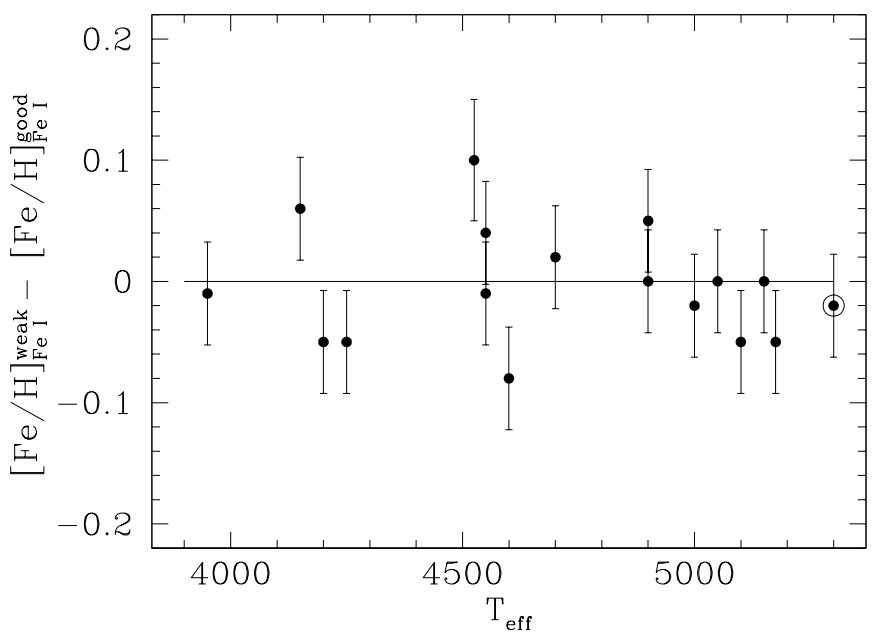

FIG. 5.-Difference between $[\mathrm{Fe} / \mathrm{H}]$ computed with the derived $\xi$ for the set of weak $\mathrm{Fe}$ I lines and with $\xi$ from the set of all good $\mathrm{Fe}$ I lines plotted with respect to $T_{\text {eff }}$. The solid line indicates equality. The only RHB star for which $\xi$ can be determined spectroscopically is marked with an open circle. 


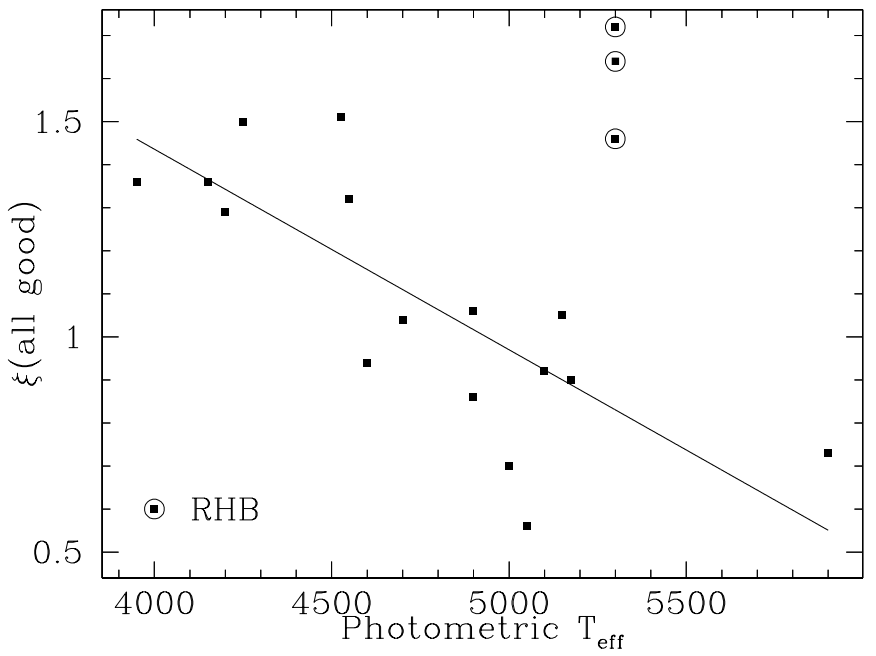

FIG. 6.- $\xi$ determined for the set of all good Fe I lines shown as a function of $T_{\text {eff }}$. The solid line is the linear fit weighted by the errors. The RHB stars are excluded from the fit and are marked with circles. The scatter around the solid line is about $0.2 \mathrm{~km} \mathrm{~s}^{-1}$.

for the weak-line set of $\mathrm{Fe} \mathrm{I}$ lines are listed in Table 1. Only 18 of our stars have enough weak $\mathrm{Fe}$ I lines to derive $\xi$ spectroscopically. We also compute $\xi$ using all the good $\mathrm{Fe}$ I lines for 20 of our stars. The difference of the resulting $[\mathrm{Fe} / \mathrm{H}]$ computed with the derived $\xi$ for the two set of lines is plotted with respect to $T_{\text {eff }}$ in Figure 5 . The mean difference is $-0.004 \pm 0.011$; hence, as expected, the $[\mathrm{Fe} / \mathrm{H}]$ results from both sets of lines show a very good agreement.

The relationship between $\xi$ determined for the set of all good $\mathrm{Fe}$ I lines and the photometric $T_{\text {eff }}$ is shown in Figure 6 . The solid line corresponds to a linear least-squares fit of the data, excluding the red horizontal branch (RHB) stars, marked with circles. The best-fit line is given by

$$
\xi=3.30-4.66 \times 10^{-4} \times T_{\text {eff }} .
$$

The scatter around the solid line is about $0.2 \mathrm{~km} \mathrm{~s}^{-1}$, which is a reasonable estimation of the error in $\xi$. For the rest of the analysis, we will use the set of all the good $\mathrm{Fe}$ I and $\mathrm{Fe}$ II lines, with $\xi$ computed from the $\xi$ - $T_{\text {eff }}$ fit. For the RHB stars we use a value of $1.61 \mathrm{~km} \mathrm{~s}^{-1}$, which corresponds to the mean value determined for the three RHB stars. The microturbulent velocity used for our stars is listed in Table 1.

\section{RESULTS}

Given the stellar parameters from Table 1, we determined the iron abundance using the equivalent widths of all the good $\mathrm{Fe} \mathrm{I}$ and $\mathrm{Fe}$ II lines identified in the HIRES spectra. We employ the grid of stellar atmospheres from Kurucz (1993b) to compute the iron abundance using the four stellar atmosphere models with the closest $T_{\text {eff }}$ and $\log (g)$ to each star's parameters. The $[\mathrm{Fe} / \mathrm{H}]$ listed in Table 1 is an interpolation of the results from the closest stellar model atmospheres to the appropriate $T_{\text {eff }}$ and $\log (g)$ for each star.

\section{1. $[\mathrm{Fe} / \mathrm{H}]$ from $\mathrm{Fe}$ I lines}

The results of $[\mathrm{Fe} / \mathrm{H}]$ from $\mathrm{Fe}$ I lines are listed in column (9) of Table 1 and plotted against the photometric $T_{\text {eff }}$ in Figure 7 (top). $T_{\text {eff }}$ is used for the x-axis as a convenient parameter for characterizing the position of the stars in the color-magnitude diagram as it also ranks the stars in lumi-
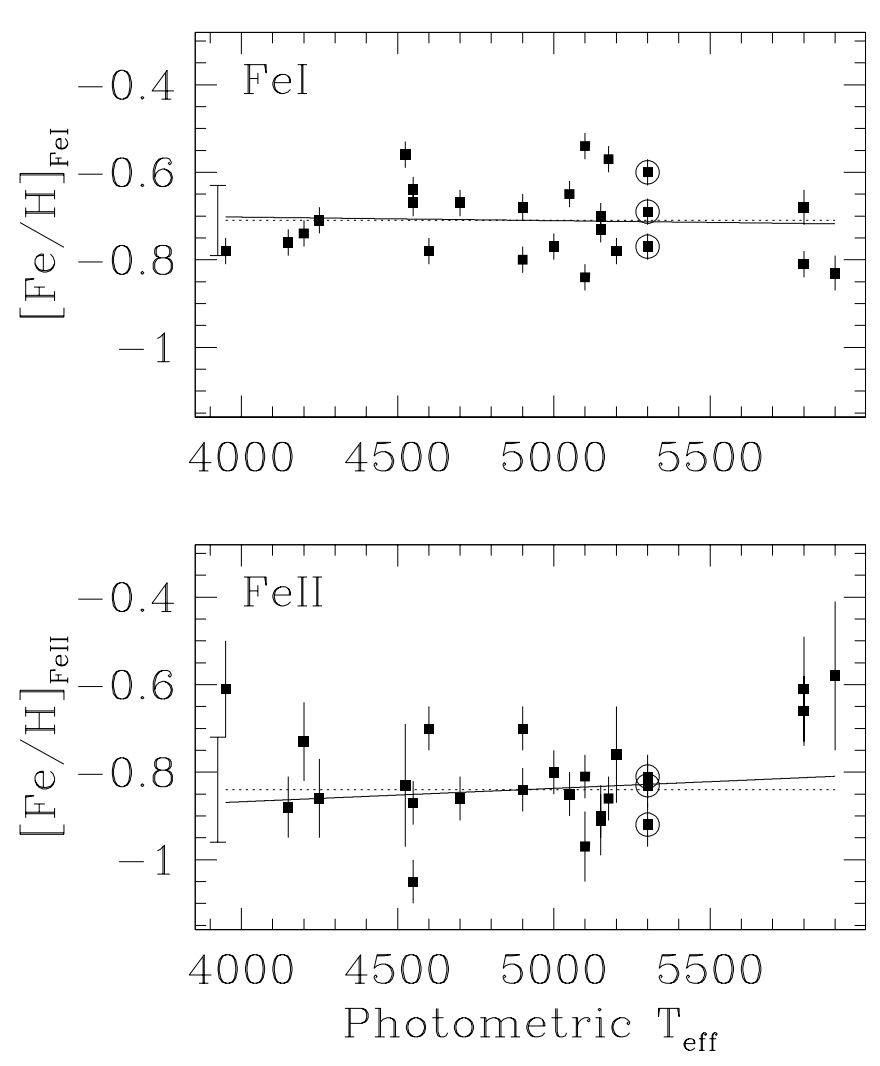

FIG. 7.- $[\mathrm{Fe} / \mathrm{H}]$ from $\mathrm{Fe}$ I (top) and $\mathrm{Fe}$ II (bottom) against photometric $T_{\text {eff }}$. The solid lines are linear fits weighted by the errors. In both cases [Fe/H] shows no dependence with $T_{\text {eff }}$. The dashed lines indicate the mean $[\mathrm{Fe} / \mathrm{H}]$ with their respective error plotted as an error bar at $3925 \mathrm{~K}$. Note that $\left\langle[\mathrm{Fe} / \mathrm{H}]_{\mathrm{Fe} \text { I }}\right\rangle=-0.71 \pm 0.08$ and $\left\langle[\mathrm{Fe} / \mathrm{H}]_{\mathrm{Fe} \mathrm{II}}\right\rangle=-0.84 \pm 0.12$. The RHB stars are marked with a open circles.

nosity (except for the RHB stars). The errors listed in Table 1 correspond to the larger of the statistical uncertainty, given by the standard deviation of the iron abundance from different lines divided by the square root of the number of lines, or a minimum value of 0.03 dex. These errors are lower limits to the actual uncertainties in the abundances, since they do not include uncertainties due to the stellar parameters nor any systematic effects that might be present. We estimate the sensitivity of $[\mathrm{Fe} / \mathrm{H}]$ derived from $\mathrm{Fe}$ I lines with respect to the stellar parameters in three cases 4250 / $1.0 / 1.0,5000 / 2.5 / 1.0$, and 5500/4.0/1.0, where the three numbers correspond to $T_{\text {eff }} / \log (g) / \xi$. The results are listed in Table 2, where the range adopted for each parameter is representative of its uncertainty. Our determination of $[\mathrm{Fe} / \mathrm{H}]$ from $\mathrm{Fe}$ I lines is most sensitive to errors in $T_{\text {eff }}$, which is less than $\sim 0.1$ dex for $\Delta T_{\text {eff }}$ of $\pm 100 \mathrm{~K}$ and have a minimal sensitivity on the choice of metallicity of the model

TABLE 2

Sensitivity OF $[\mathrm{Fe} / \mathrm{H}]_{\mathrm{Fe} \text { I }}$ ON STEllar Parameters

\begin{tabular}{ccccc}
\hline \hline & $\Delta T_{\text {eff }}$ & $\Delta \log (g)$ & $\Delta \xi$ & $\Delta[\mathrm{Fe} / \mathrm{H}]$ \\
$T_{\text {eff }} / \log (g) / \xi$ & $(+100 \mathrm{~K})$ & $(+0.2 \mathrm{dex})$ & $\left(+0.2 \mathrm{~km} \mathrm{~s}^{-1}\right)$ & $(+0.2 \mathrm{dex})$ \\
\hline $4250 / 1.0 / 1.0 \ldots \ldots$ & +0.04 & +0.02 & $-0.08^{\mathrm{a}}$ & -0.03 \\
$5000 / 2.5 / 1.0 \ldots \ldots$ & +0.09 & +0.01 & $-0.06^{\mathrm{a}}$ & -0.01 \\
$5500 / 4.0 / 1.0 \ldots \ldots$ & +0.08 & +0.02 & $-0.03^{\mathrm{a}}$ & -0.01 \\
\hline
\end{tabular}

${ }^{\text {a }}$ This is for the set of good Fe I lines. It is smaller by a factor of 3 for the set of weak Fe I lines. 
TABLE 3

Sensitivity of $[\mathrm{Fe} / \mathrm{H}]_{\mathrm{Fe} \mathrm{II}}$ ON Stellar Parameters

\begin{tabular}{ccccc}
\hline \hline & $\Delta T_{\text {eff }}$ & $\Delta \log (g)$ & $\Delta \xi$ & $\Delta[\mathrm{Fe} / \mathrm{H}]$ \\
$T_{\text {eff }} / \log (g) / \xi$ & $(+100 \mathrm{~K})$ & $(+0.2 \mathrm{dex})$ & $\left(+0.2 \mathrm{~km} \mathrm{~s}^{-1}\right)$ & $(+0.2 \mathrm{dex})$ \\
\hline $4250 / 1.0 / 1.0 \ldots \ldots$ & -0.12 & +0.11 & -0.04 & -0.07 \\
$5000 / 2.5 / 1.0 \ldots \ldots$ & -0.02 & +0.09 & -0.03 & -0.04 \\
$5500 / 4.0 / 1.0 \ldots \ldots$ & -0.03 & +0.08 & -0.02 & -0.03 \\
\hline
\end{tabular}

atmosphere grid for plausible changes in $[\mathrm{Fe} / \mathrm{H}]( \pm 0.2$ dex).

The solid line shown in Figure 7 (top) is a linear fit weighted by the errors of $[\mathrm{Fe} / \mathrm{H}]$ versus $T_{\text {eff }}$. The slope of the fit is $(-0.8 \pm 3.6) \times 10^{-5}$ dex $\mathrm{K}^{-1}$, which is consistent with $[\mathrm{Fe} / \mathrm{H}]$ being constant, independent of $T_{\text {eff }}$ (i.e., of luminosity or equivalently position in the color-magnitude diagram). We divide our sample in four groups of stars: giant stars at or above the RHB, stars on the RHB, giant stars below the RHB, and stars near the turnoff. The mean $[\mathrm{Fe} / \mathrm{H}]$ for each group is listed on Table 4 . We found no significant difference in the mean $[\mathrm{Fe} / \mathrm{H}]$ obtained from $\mathrm{Fe}$ I lines among the defined groups of stars.

The mean $[\mathrm{Fe} / \mathrm{H}]$ weighted by the errors of all 25 stars is $-0.71 \pm 0.08$, in very good agreement with earlier determinations (Cohen 1983; Gratton et al. 1986; Leep et al. 1987; Sneden et al. 1994).

\section{2. $[\mathrm{Fe} / \mathrm{H}]$ from $\mathrm{Fe}$ II lines}

The determinations of $[\mathrm{Fe} / \mathrm{H}]$ from $\mathrm{Fe}$ II lines are listed in column (11) of Table 1 and plotted against the photo-

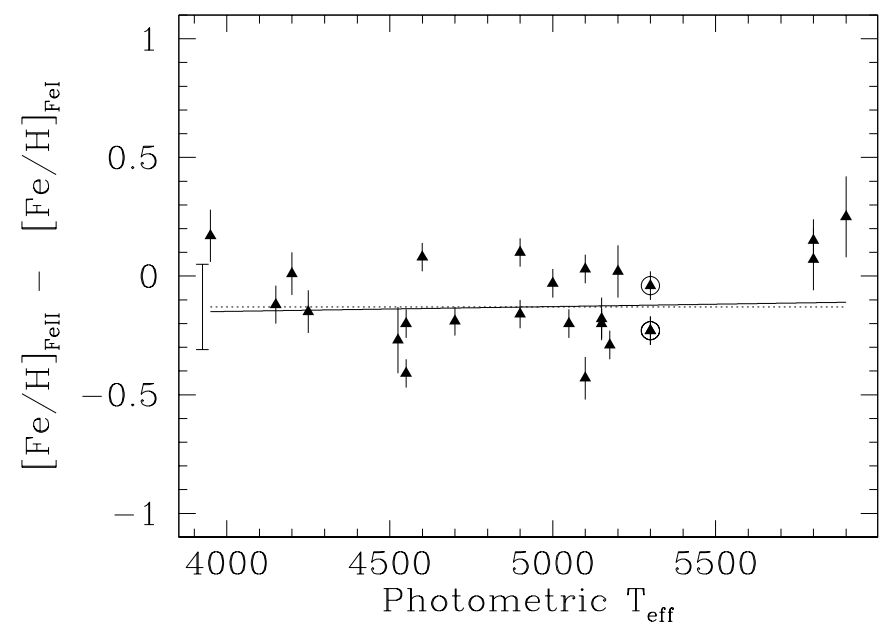

FIG. 8.-Difference between $[\mathrm{Fe} / \mathrm{H}]$ from $\mathrm{Fe}$ I and $\mathrm{Fe}$ II against $T_{\text {eff }}$. The solid line, which is nearly flat, is a linear fit weighted by the errors. The dashed line indicates the mean difference with its respective error plotted as an error bar at $3925 \mathrm{~K}$. Note that the mean difference is $-0.13 \pm 0.18$. The RHB stars are marked with a open circles. metric $T_{\text {eff }}$ in Figure 7 (bottom). The errors listed in Table 1 correspond to the statistical uncertainty or a value of 0.05 dex, whichever is larger. We estimate the sensitivity of $[\mathrm{Fe} / \mathrm{H}]$ derived from $\mathrm{Fe}$ II lines with respect to the stellar parameters in the same manner as the sensitivity of $[\mathrm{Fe} / \mathrm{H}]$ from $\mathrm{Fe}$ I lines. The results are listed in Table 3, where the range adopted for each parameter is representative of its uncertainty. We see a stronger sensitivity on the stellar parameters from the $\mathrm{Fe}$ II lines than from the $\mathrm{Fe}$ I lines. The $[\mathrm{Fe} / \mathrm{H}]$ determination from $\mathrm{Fe}$ II lines is most sensitive to the systematic error (note that the internal uncertainty in $\log (g)$ is $\leq 0.1$ dex) in $\log (g)$, as well as to $T_{\text {eff }}$ among the coolest M71 giants. The sensitivity on the choice of metallicity of the model atmosphere grid is small for reasonable changes in metallicity.

The solid line shown in Figure 7 (bottom) is a linear fit weighted by the errors of $[\mathrm{Fe} / \mathrm{H}]$ versus $T_{\text {eff }}$. The slope of the fit is $(+3.1 \pm 5.2) \times 10^{-5}$ dex $\mathrm{K}^{-1}$, which is consistent with $[\mathrm{Fe} / \mathrm{H}]$ being constant, independent of $T_{\text {eff }}$. We found that there is no significant difference in the mean $[\mathrm{Fe} / \mathrm{H}]$ obtained from $\mathrm{Fe}$ II among stars from different luminosity groups, listed in Table 4 . The mean $[\mathrm{Fe} / \mathrm{H}]$ weighted by the errors is $-0.84 \pm 0.12$, in very good agreement with our result from $\mathrm{Fe} \mathrm{I}$ lines and earlier determinations (Cohen 1983; Gratton et al. 1986; Leep et al. 1987; Sneden et al. 1994).

\subsection{Non-LTE Effects}

The iron abundance could be affected by departures from LTE. The main non-LTE (NLTE) effect in late-type stars is caused by overionization of electron donor metals by ultraviolet radiation (Auman \& Woodrow 1975). Recently, Gratton et al. (1999) and Thévenin \& Idiart (1999) studied NLTE effects in Fe abundances in metal-poor late-type stars. Gratton et al. (1999) found that NLTE corrections for $\mathrm{Fe}$ lines are very small in dwarfs of any $T_{\text {eff }}$, and only small corrections $(<0.1 \mathrm{dex})$ are expected for stars on the red giant branch. Thévenin \& Idiart (1999) found that NLTE corrections become less important as $[\mathrm{Fe} / \mathrm{H}]$ increases, being less than 0.1 dex for stars with $[\mathrm{Fe} / \mathrm{H}]>-0.75$ dex, and that ionized lines are not significantly affected by NLTE.

One way to explore possible NLTE effects present in our data is by comparing the results from $\mathrm{Fe} \mathrm{I}$ and $\mathrm{Fe}$ II lines. The difference between $[\mathrm{Fe} / \mathrm{H}]$ from $\mathrm{Fe}$ II and $\mathrm{Fe} \mathrm{I}$ lines is plotted in Figure 8 against $T_{\text {eff }}$. The solid line is a linear fit weighted by the errors. The slope of the fit is $(+2.0 \pm 8.2) \times 10^{-5}$ dex $\mathrm{K}^{-1}$, which is nearly flat. The mean difference is $-0.13 \pm 0.18$. We conclude that NLTE effects are negligible in our iron abundance determination, as expected from results of earlier studies (Gratton et al. 1999; Thévenin \& Idiart 1999).

TABLE 4

$[\mathrm{Fe} / \mathrm{H}]$ FOR EACH GROUP OF Stars

\begin{tabular}{|c|c|c|c|c|}
\hline Star Group & $\langle V\rangle$ & $N_{\text {stars }}$ & $\left\langle[\mathrm{Fe} / \mathrm{H}]_{\mathrm{Fe} \mathbf{I}}\right\rangle$ & $\left\langle[\mathrm{Fe} / \mathrm{H}]_{\mathrm{Fe} \mathrm{II}}\right\rangle$ \\
\hline RGB at or above RHB. & 13.46 & 10 & $-0.71 \pm 0.07$ & $-0.83 \pm 0.12$ \\
\hline RHB . & 14.50 & 3 & $-0.68 \pm 0.07$ & $-0.86 \pm 0.05$ \\
\hline RGH below RHB ............ & 15.92 & 9 & $-0.69 \pm 0.09$ & $-0.85 \pm 0.06$ \\
\hline Near TO ..................... & 17.76 & 3 & $-0.78 \pm 0.06$ & $-0.64 \pm 0.13$ \\
\hline
\end{tabular}




\section{DISCUSSION}

Our $[\mathrm{Fe} / \mathrm{H}]$ abundance results involve a wide luminosity sample of stars, which includes at the same time stars from luminous giants to stars near the turnoff. We find that the $[\mathrm{Fe} / \mathrm{H}]$ abundance, from both $\mathrm{Fe} \mathrm{I}$ and $\mathrm{Fe}$ II lines, is independent of $T_{\text {eff }}$ and equivalently luminosity.

Our result is in agreement with the work of Gratton et al. (2001). They present abundances from high-dispersion spectra from the VLT of stars in NGC 6397 and NGC 6752. They found that the $[\mathrm{Fe} / \mathrm{H}]$ obtained for stars at the base of the subgiant branch agrees within a few percent with the $[\mathrm{Fe} / \mathrm{H}]$ obtained for stars at the main sequence turnoff and further compare this value with analysis of the RGB stars in this cluster by other groups. Note that the luminosity range of the sample presented in our work is several orders of magnitude wider than the luminosity range of Gratton et al.'s (2001) sample.

Our results, and those of Gratton et al. (2001), appear to be in disagreement with inhomogeneities in $[\mathrm{Fe} / \mathrm{H}]$ found earlier by King et al. (1998). They obtained $[\mathrm{Fe} / \mathrm{H}]=$ -2.52 dex for a sample of subgiant stars in M92, which is a factor of 2 smaller than $[\mathrm{Fe} / \mathrm{H}]$ measurements using red giants in the same cluster (Cohen 1978; Sneden et al. 1991). King et al. (1998) compare their result for the M92 subgiants with analysis of RBG stars by other groups, who may have determined the stellar parameters and performed the abundance determinations in a different way. This possible difference in the analysis of the giant and subgiant sample may account for the difference in $[\mathrm{Fe} / \mathrm{H}]$ found by King et al. (1998), or perhaps the determination of the stellar parameters by King et al. (1998) is flawed. Our result, on the other hand, is robust, because we have determined both the stellar parameters and the $\mathrm{Fe}$ abundance in a homogeneous and consistent manner for all our stars.

Gratton et al. (2001) also found that NGC 6397 is homogeneous in both $\mathrm{O}$ and $\mathrm{Fe}$, while an $\mathrm{O}-\mathrm{Na}$ anticorrelation is present among unevolved stars in NGC 6752, which is very difficult to explain by the deep mixing scenario. Lines from many additional species, including $\mathrm{O}, \mathrm{Na}, \mathrm{Mg}, \mathrm{Ti}$, and $\mathrm{Sc}$ among others, are observed in our HIRES spectra. We plan to present additional information in the matter of light, iron-peak, and heavy elements in the near future.

\section{CONCLUSIONS}

We present results of a high-dispersion analysis of $\mathrm{Fe} I$ and $\mathrm{Fe}$ II lines to obtain $[\mathrm{Fe} / \mathrm{H}]$ for 25 members of the Galactic globular cluster M71. Our sample of stars includes 19 giant stars, three horizontal branch stars, and three stars near the turnoff. Our conclusions are summarized as follows:

1. The $[\mathrm{Fe} / \mathrm{H}]$ obtained from $\mathrm{Fe}$ I lines agrees very well with the $[\mathrm{Fe} / \mathrm{H}]$ obtained from $\mathrm{Fe}$ II lines.

2. The mean $[\mathrm{Fe} / \mathrm{H}]$ obtained from $\mathrm{Fe}$ I and $\mathrm{Fe}$ II lines of all 25 stars is in good agreement with earlier determinations.

3. The $[\mathrm{Fe} / \mathrm{H}]$ obtained from both $\mathrm{Fe}$ I and $\mathrm{Fe}$ II lines is independent of $T_{\text {eff }}$ and equivalently luminosity.

4. No difference is found among the mean $[\mathrm{Fe} / \mathrm{H}]$ from giant stars located at or above the RHB, RHB stars, giant stars located below the RHB, and stars near the mainsequence turnoff.

In the near future, we will present the result from the analysis now underway of additional elements.

The entire Keck/HIRES and LRIS user community owes a huge debt to Jerry Nelson, Gerry Smith, Steve Vogt, Bev Oke, and many other people who have worked to make the Keck telescope and HIRES and LRIS a reality and to operate and maintain the Keck Observatory. We are grateful to the W. M. Keck Foundation for the vision to fund the construction of the W. M. Keck Observatory. We thank R. Gratton for providing a detailed description of his automatic equivalent width measuring program and R. Pogge for providing the Gaussian profile-fitting routine. Partial support to M. M. B. was provided by a Theodore Dunham, Jr., grant for research in astronomy and by the National Science Foundation under grant AST 96-24680 to M. M. B. and grant AST 98-19614 to J. G. C.

\section{APPENDIX A}

\section{ERRORS IN THE EQUIVALENT WIDTHS}

The equivalent width of a line is defined as

$$
W_{\lambda}=\int \frac{g(\lambda)}{\operatorname{cont}(\lambda)} d \lambda=\sum_{i} \frac{g\left(\lambda_{i}\right)}{\operatorname{cont}\left(\lambda_{i}\right)} \Delta \lambda,
$$

where, $g(\lambda)$ is the flux of the spectrum, $\operatorname{cont}(\lambda)$ is the continuum level, and $\Delta \lambda$ is the step that can be made arbitrarily small to better approximate the integral. In this case, $g(\lambda)$ is the Gaussian profile of the line, given by

$$
g(\lambda)=P \times \exp \left[\frac{-\left(\lambda-\lambda_{\text {cen }}\right)^{2}}{2 \sigma^{2}}\right],
$$

where $P$ is the peak of the Gaussian, $\lambda_{\text {cen }}$ is the central wavelength, and $\sigma$ is the dispersion of the Gaussian.

If the equivalent width is computed through out the summation, then the error in the equivalent width $\sigma_{\mathrm{w}}$ will be the quadratic summation of the errors in the individual points times the step

$$
\sigma_{W_{\lambda}}^{2}=\sum_{i}\left(\sigma_{i} \times \Delta \lambda\right)^{2},
$$


where

$$
\begin{gathered}
\sigma_{i}^{2}=\left(\frac{\partial w}{\partial g} \sigma_{g i}\right)^{2}+\left(\frac{\partial w}{\partial \text { cont }} \sigma_{\text {cont }}\right)^{2} \\
\sigma_{i}^{2}=\left[\frac{1}{\operatorname{cont}\left(\lambda_{i}\right)} \sigma_{g i}\right]^{2}+\left[\frac{g\left(\lambda_{i}\right)}{\operatorname{cont}\left(\lambda_{i}\right)^{2}} \sigma_{\text {cont }}\right]^{2} .
\end{gathered}
$$

If we assume that the continuum level is equal to 1 , then

$$
\sigma_{i}^{2}=\sigma_{g i}^{2}+g\left(\lambda_{i}\right)^{2} \sigma_{\text {cont }}^{2} .
$$

Since the Gaussian depends on three parameters:

$$
\begin{gathered}
\sigma_{g i}^{2}=\left(\frac{\partial g}{\partial P} \sigma_{P}\right)^{2}+\left(\frac{\partial g}{\partial \lambda_{\mathrm{cen}}} \sigma_{\lambda_{\mathrm{cen}}}\right)^{2}+\left(\frac{\partial g}{\partial \sigma} \sigma_{\sigma}\right)^{2} \\
\sigma_{g i}^{2}=\left[\frac{g\left(\lambda_{i}\right)}{P} \sigma_{P}\right]^{2}+\left[\frac{\left(\lambda_{i}-\lambda_{\mathrm{cen}}\right)}{\sigma^{2}} g\left(\lambda_{i}\right) \sigma_{\lambda_{\mathrm{cen}}}\right]^{2}+\left[\frac{\left(\lambda_{i}-\lambda_{\mathrm{cen}}\right)^{2}}{\sigma^{3}} g\left(\lambda_{i}\right) \sigma_{\sigma}\right]^{2} \\
\sigma_{g i}^{2}=g\left(\lambda_{i}\right)^{2} \times\left[\frac{\sigma_{P}^{2}}{P^{2}}+\frac{\left(\lambda_{i}-\lambda_{\mathrm{cen}}\right)^{2}}{\sigma^{4}} \sigma_{\lambda_{\mathrm{cen}}}^{2}+\frac{\left(\lambda_{i}-\lambda_{\mathrm{cen}}\right)^{4}}{\sigma^{6}} \sigma_{\sigma}^{2}\right]
\end{gathered}
$$

Finally,

$$
\sigma_{i}^{2}=g\left(\lambda_{i}\right)^{2} \times\left[\frac{\sigma_{P}^{2}}{P^{2}}+\frac{\left(\lambda_{i}-\lambda_{\mathrm{cen}}\right)^{2}}{\sigma^{4}} \sigma_{\lambda_{\mathrm{cen}}}^{2}+\frac{\left(\lambda_{i}-\lambda_{\mathrm{cen}}\right)^{4}}{\sigma^{6}} \sigma_{\sigma}^{2}+\sigma_{\mathrm{cont}}^{2}\right] .
$$

Arp, H. C., \& Hartwick, F. D. A. 1971, ApJ, 167, 499

Auman, J. R., \& Woodrow, J. E. J. 1975, ApJ, 197, 163

Bard, A., Kock, A., \& Kock, M. 1991, A\&A, 248, 315

Bard, A., \& Kock, M. 1994, A\&A, 282, 1014

Biémont, E., Baudoux, M., Kurucz, R. L., Ansbacher, W., \& Pinnington, E. H. 1991, A\&A, 249, 539

Blackwell, D. E., Booth, A. J., Haddock, D. J., Petford, A. D., \& Leggett, S. K. 1986, MNRAS, 220, 549

Blackwell, D. E., Petford, A. D., \& Shallis, M. J. 1979, MNRAS, 186, 657

Blackwell, D. E., Petford, A. D., Shallis, M. J., \& Simmons, G. J. 1982a, MNRAS, 199, 43

Blackwell, D. E., Petford, A. D., \& Simmons, G. J. 1982b, MNRAS, 201, 595

Blackwell, D. E., Shallis, M. J., \& Simmons, G. J. 1980, A\&A, 81, 340

Castilho, B. V., Pasquini, L., Allen, D. M., Barbuy, B., \& Molaro, P. 2000, A\&A, 361, 92

Cohen, J. G. 1978, ApJ, 223, 487 .1983, ApJ, 270, 654

Cohen, J. G., Briley, M. M., \& Behr, B. B. 2001, AJ, 122, 1420 (Paper I)

Gratton, R. G., Quarta, M. L., \& Ortolani, S. 1986, A\&A, 169, 208

Gratton, R. G., et al. 2001, A\&A, 369, 87

Gratton, R. G., Carretta, E., Eriksson, K., \& Gustafsson, B. 1999, A\&A, 350,955

\section{REFERENCES}

Holweger, H., Bard, A., Kock, A., \& Kock, M. 1991, A\&A, 249, 545

King, J. R., Stephens, A., Boesgaard, A. M., \& Deliyannis, C. P. 1998, AJ, 115,666

Kurucz, R. L. 1993a, CD-ROM No. 13, ATLAS9 Stellar Atmosphere Programs and $2 \mathrm{~km} / \mathrm{s}$ Grid (Cambridge: Smithsonian Astrophys. Obs.) . 1993b, CD-ROM No. 18, SYNTHE Spectrum Synthesis Programs and Line Data (Cambridge: Smithsonian Astrophys. Obs.)

Lambert, D. L., Heath, J. E., Lemke, M., \& Drake, J. 1996, ApJS, 103, 183

Leep, E. M., Oke, J. B., \& Wallerstein, G. 1987, AJ, 93, 338

May, M., Richter, J., \& Wichelmann, J. 1974, A\&AS, 18, 405

O'Brian, T. R., Wickliffe, M. E., Lawler, J. E., Whaling, W., \& Brault, J. W. 1991, J. Opt. Soc. Am., B8, 1185

Sneden, C. 1973, Ph.D. thesis, Univ. Texas

Sneden, C., Kraft, R. P., Langer, G. E., Prosser, C. F., \& Shetrone, M. D. 1994, AJ, 107, 1773

Sneden, C., Kraft, R. P., Prosser, C. F., \& Langer, G. E. 1991, AJ, 102, 2001

Thévenin, F. 1989, A\&AS, 77, 137

. 1990, A\&AS, 82, 179

Thévenin, F., \& Idiart, T. P. 1999, ApJ, 521, 753

Wolnik, S. J., Berthel, R. O., \& Wares, G. W. 1971, ApJ, 166, L31 